Supporting Information for:

"Searching the force field electrostatic multipole parameter space"

\author{
Sofie Jakobsen and Frank Jensen* \\ Department of Chemistry, Aarhus University \\ DK-8000 Aarhus, Denmark \\ * Corresponding author
}




\section{Figure SI.1}

Atomic labeling for CYS, which is used in the following tables.

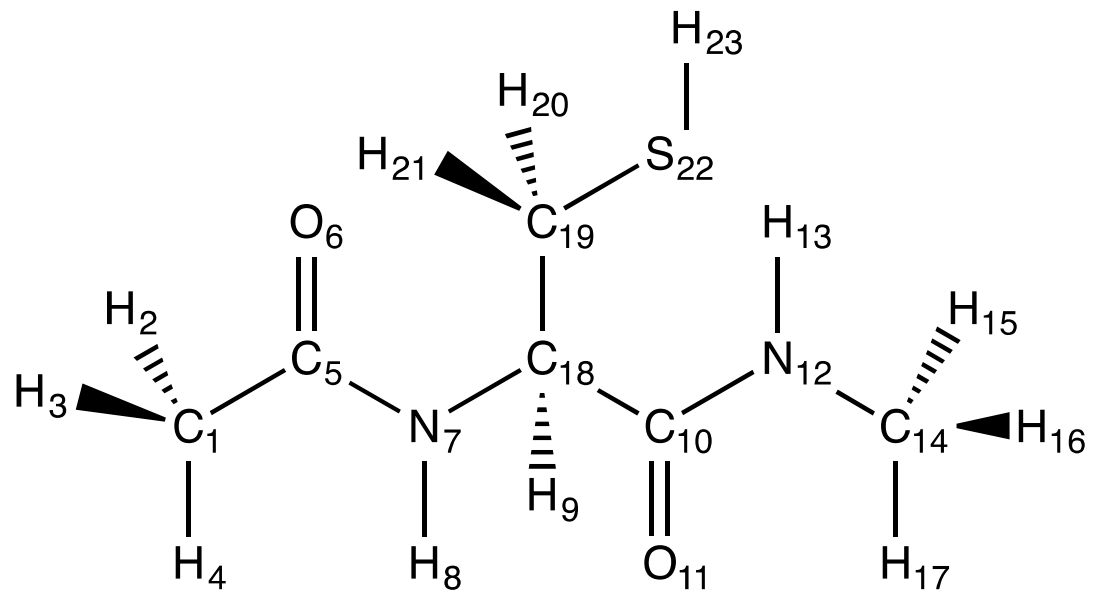




\section{Table SI.1}

Atomic coordinates in units of Ångstrøm for CYS. The structure is visualized in Figure 3.b in the paper.

\begin{tabular}{lrrr}
\hline Atom & $\mathrm{x}$ & $\mathrm{y}$ & $\mathrm{z}$ \\
\hline $\mathrm{C}_{1}$ & -2.5297 & 0.1082 & 1.1953 \\
$\mathrm{H}_{2}$ & -2.0582 & -0.5059 & 1.9713 \\
$\mathrm{H}_{3}$ & -3.5844 & 0.2520 & 1.4312 \\
$\mathrm{H}_{4}$ & -2.0172 & 1.0772 & 1.1965 \\
$\mathrm{C}_{5}$ & -2.4330 & -0.5500 & -0.1589 \\
$\mathrm{O}_{6}$ & -3.4079 & -0.8113 & -0.8387 \\
$\mathrm{~N}_{7}$ & -1.1686 & -0.8316 & -0.6253 \\
$\mathrm{H}_{8}$ & -1.1696 & -1.3608 & -1.4873 \\
$\mathrm{H}_{9}$ & -0.0201 & -1.1954 & 1.1071 \\
$\mathrm{C}_{10}$ & 0.4775 & 0.7295 & 0.4147 \\
$\mathrm{O}_{11}$ & 0.9871 & 1.0297 & 1.4799 \\
$\mathrm{~N}_{12}$ & 0.2509 & 1.6058 & -0.5893 \\
$\mathrm{H}_{13}$ & -0.3332 & 1.2978 & -1.3522 \\
$\mathrm{C}_{14}$ & 0.5547 & 3.0146 & -0.4489 \\
$\mathrm{H}_{15}$ & 1.5018 & 3.1226 & 0.0850 \\
$\mathrm{H}_{16}$ & 0.6467 & 3.4633 & -1.4414 \\
$\mathrm{H}_{17}$ & -0.2232 & 3.5447 & 0.1155 \\
$\mathrm{C}_{18}$ & 0.0735 & -0.7247 & 0.1206 \\
$\mathrm{C}_{19}$ & 1.1520 & -1.4718 & -0.6748 \\
$\mathrm{H}_{20}$ & 0.8776 & -2.5311 & -0.7226 \\
$\mathrm{H}_{21}$ & 1.1838 & -1.0835 & -1.7004 \\
$\mathrm{~S}_{22}$ & 2.8578 & -1.2958 & -0.0639 \\
$\mathrm{H}_{23}$ & 2.5815 & -1.4682 & 1.2465 \\
\hline & & &
\end{tabular}




\section{Table SI.2}

Cartesian multipole moments of CYS for a reduced set of 29 parameters, obtained by the twostage approach (see Ref. 8). This set of parameters provides an ESP RMS-error of $3.59 \cdot 10^{-3} E_{\mathrm{h}} / e$. Blank entries correspond to multipole parameters that have been eliminated. All units are in atomic units, i.e., charges are in units of $e$, dipole moments in units of $e a_{0}$, and quadrupole moments in units of $e a_{0}^{2}$.

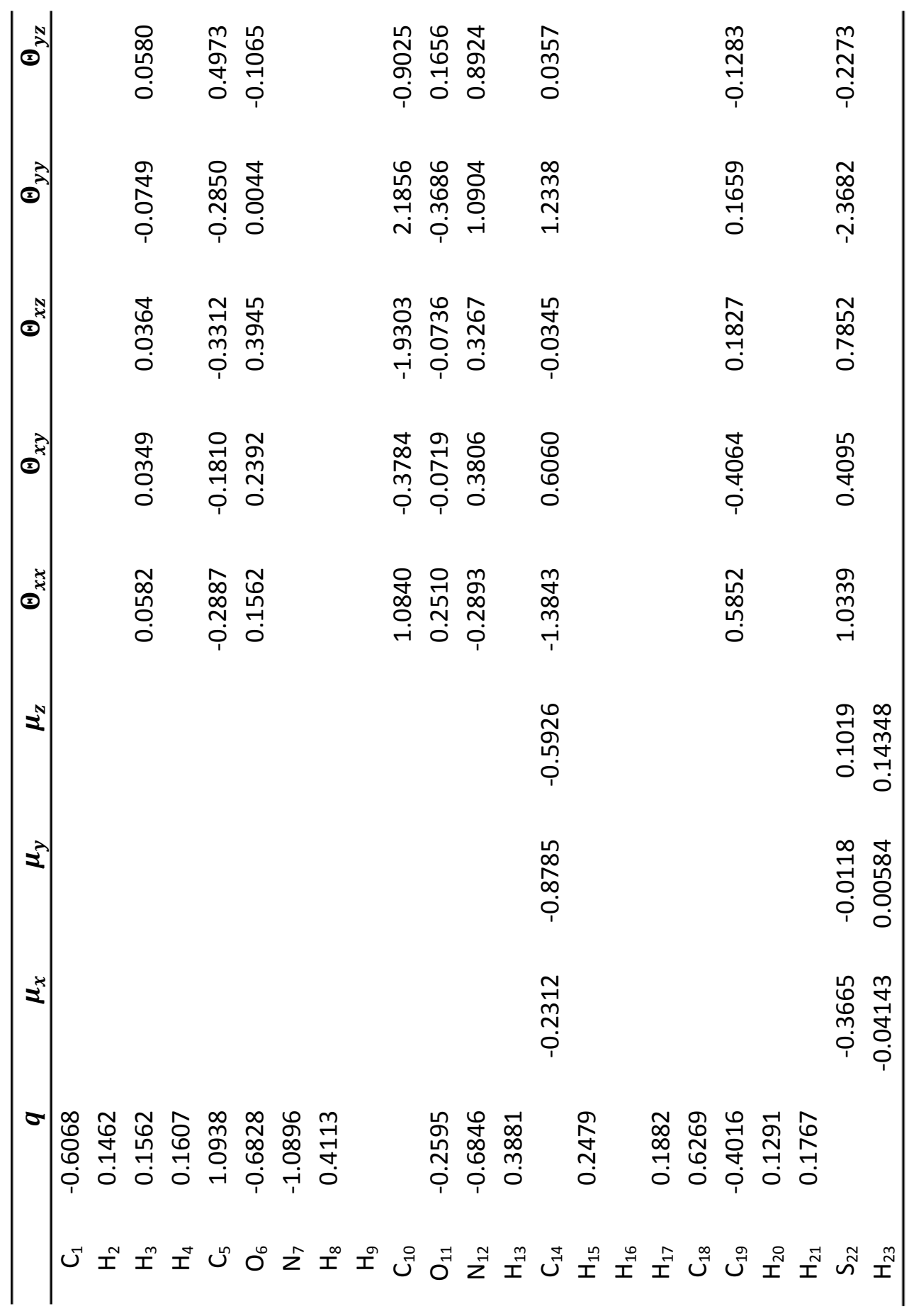




\section{Table SI.3}

Cartesian multipole moments of CYS for a reduced set of 29 parameters, obtained by the buttomup method using a weighting scheme of 1:1:1 (corresponding to the graph labeled 'Rank 1+ 1:1:1' in Figure 6 in the paper). This set of parameters provides an ESP RMS-error of $3.48 \cdot 10^{-3} E_{\mathrm{h}} / e$. Blank entries correspond to multipole parameters that have been eliminated. All units are in atomic units, i.e., charges are in units of $e$, dipole moments in units of $e a_{0}$, and quadrupole moments in units of $e a_{0}^{2}$.

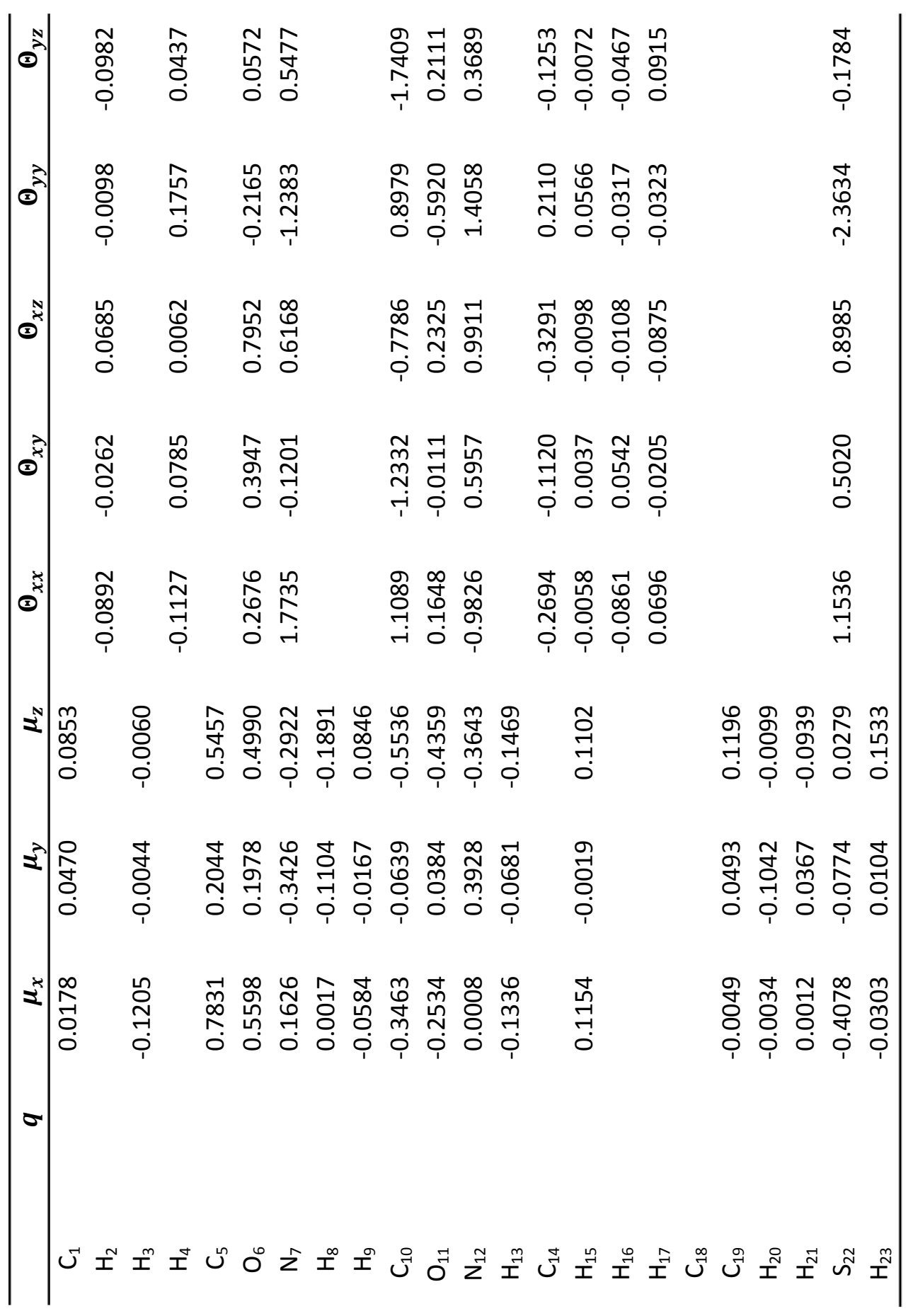




\section{Table SI.4}

Cartesian multipole moments of CYS for a reduced set of 29 parameters, obtained by the buttomup method using a weighting scheme of 1:3:5 (corresponding to the graph labeled 'Rank 1+ 1:3:5' in Figure 6 in the paper). This set of parameters provides an ESP RMS-error of $3.53 \cdot 10^{-3} E_{\mathrm{h}} / e$. Blank entries correspond to multipole parameters that have been eliminated. All units are in atomic units, i.e., charges are in units of $e$, dipole moments in units of $e a_{0}$, and quadrupole moments in units of $e a_{0}^{2}$.

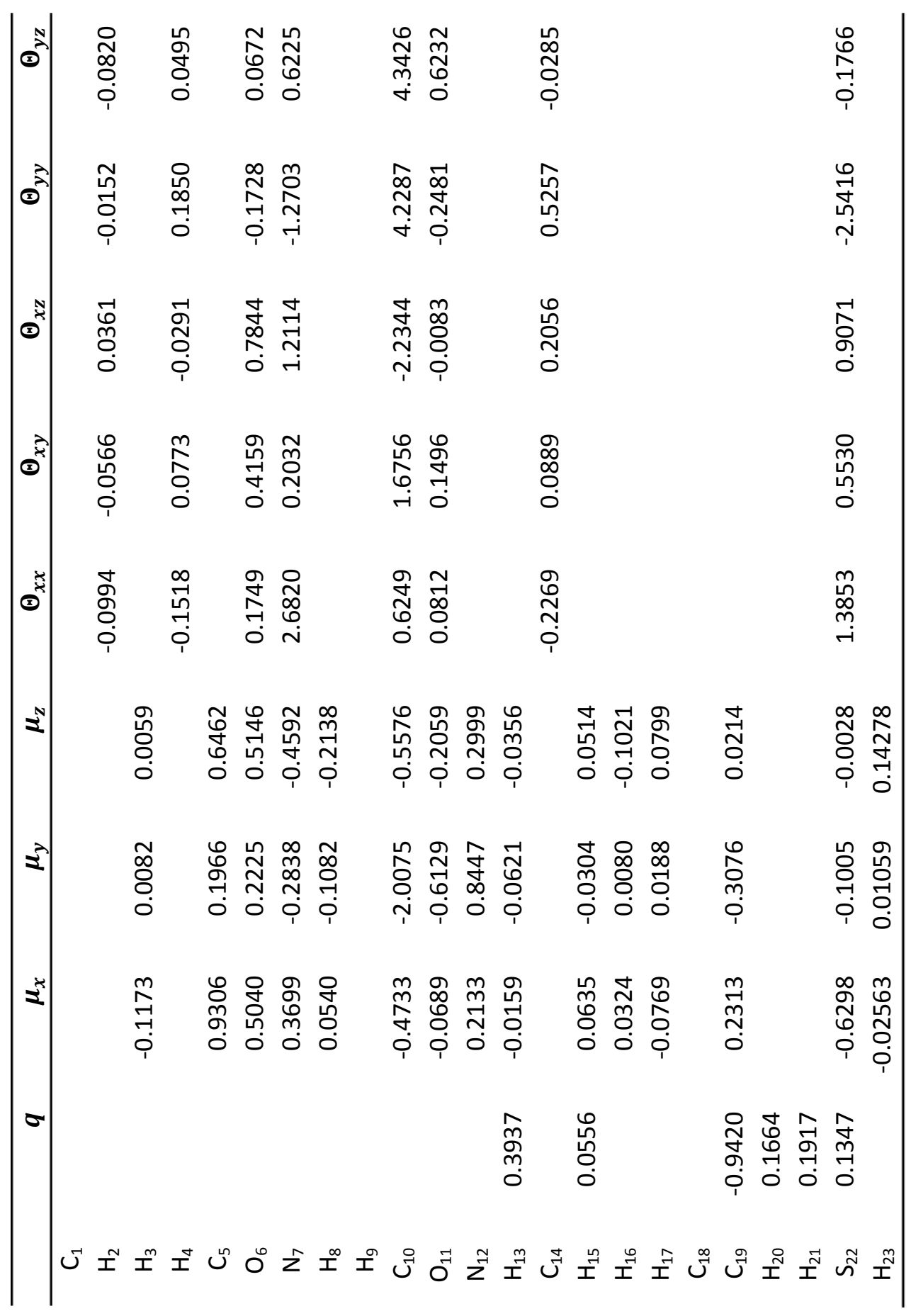




\section{Table SI.5}

Cartesian multipole moments of CYS for a reduced set of 29 parameters, obtained by the buttomup method using a weighting scheme of 1:5:25 (corresponding to the graph labeled 'Rank 1+ 1:5:25' in Figure 6 in the paper). This set of parameters provides an ESP RMS-error of $4.02 \cdot 10^{-3}$ $E_{\mathrm{h}} / e$. Blank entries correspond to multipole parameters that have been eliminated. All units are in atomic units, i.e., charges are in units of $e$, dipole moments in units of $e a_{0}$, and quadrupole moments in units of $e a_{0}^{2}$.

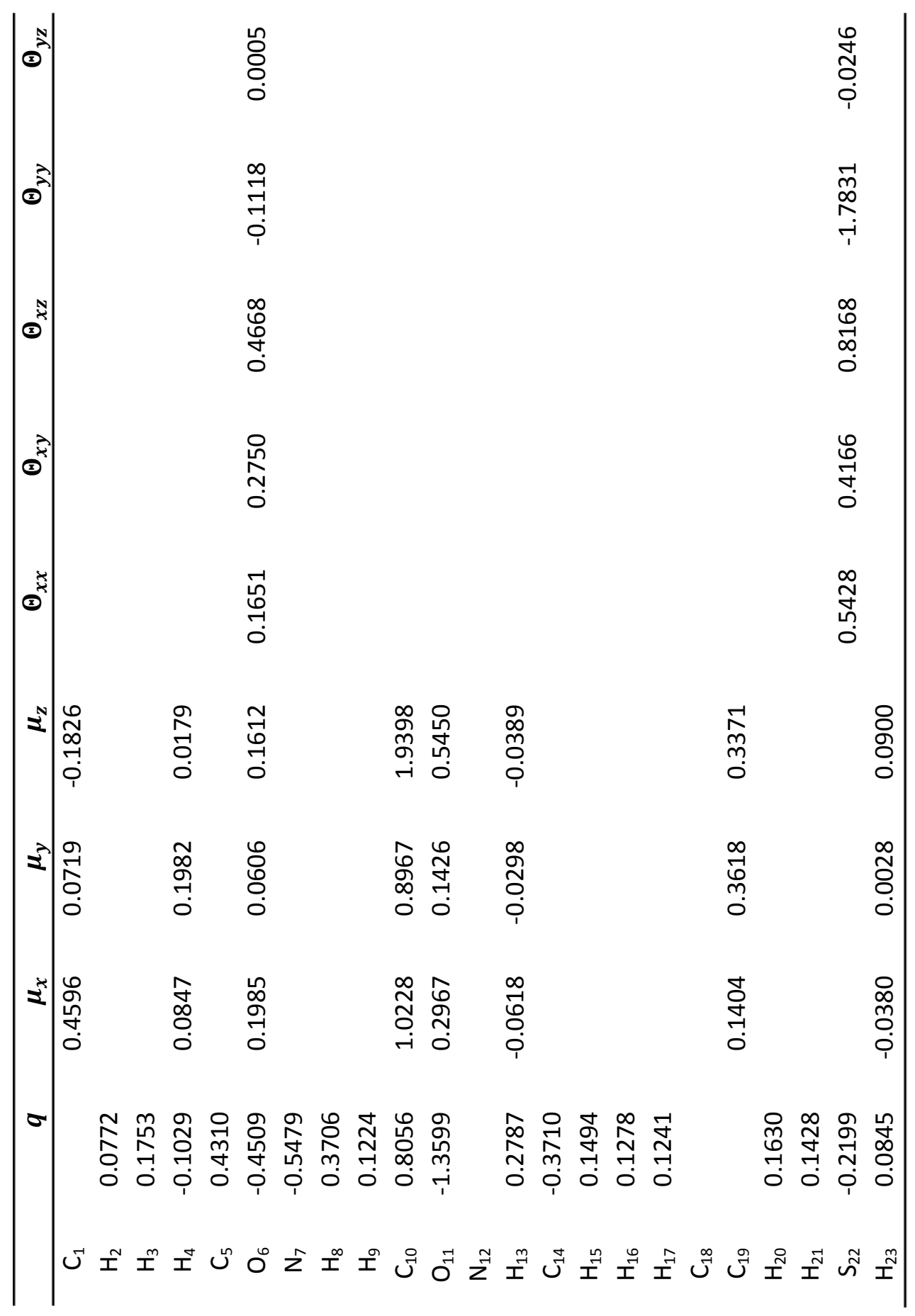

